\title{
Tumor cervical por enfermedad de Kikuchi. U na entidad clínica a la que no estamos habituados. Caso clínico
}

\author{
Patricio Gac E $\mathrm{E}^{1}$, Patricio Cabané $\mathrm{T}^{1}$, Carmen Franco $\mathrm{S}^{2}$, \\ José Amat $\mathbf{V}^{\mathbf{1}}$, Ricardo Rossi $\mathrm{F}^{\mathbf{1}}$, Felipe Huidobro $\mathrm{G}^{1}$, \\ Laura Carreño $\mathrm{T}^{1}$, Patricio Covarrubias $\mathrm{C}^{1}$. \\ Histiocytic necrotizing lymphadenitis. \\ Report of three cases
}

\begin{abstract}
Histiocytic necrotizing lymphadenitis (HNL), also known as Kikuchi's disease is a rare condition of unknown etiology. Patients present with cervical lymph node enlargement, fever and malaise. The diagnosis is made by excision biopsy. However, this entity must be distinguished from both reactive processes and malignant tumors such as lymphoma. The clinical course is self limited with spontaneous resolution within a few months. We report three patients with the disease. A 37 year-old woman with a 4 months history of a painless submaxillary mass of $2.5 \mathrm{~cm}$ diameter, attached to the deep tissues of the neck. The mass was excised and the biopsy report was HNL After 26 months of follow up, the patient is asymptomatic. A 30 year-old woman with a history of 2 months of a painless lateral cervical mass and aspiration biopsy was reported as suspicious for lymphoma. An excision biopsy was performed, that was reported as HNL. In both patients, lymphoma was ruled out by immunohistochemistry. A 33 year-old woman with a 3 weeks history of an asymptomatic lateral cervical mass. Biopsy was reported as HNL This condition must be included in the differential diagnosis of cervical asymptomatic masses. The clinician must be aware of it to avoid longterm, costly treatments (Rev Méd Chile 2008; 136: 209-16).
\end{abstract}

(Key w ords: Histiocytic necrotizing lymphadenitis; Kikuchi disease; Lymph nodes)

Recibido el 13 de noviembre, 2006. Aceptado el 11 de junio, 2007.

${ }^{1}$ Departamento de Cirugía y Anatomía Patológica, Hospital Clínico Universidad de Chile.

${ }^{2}$ Servicio de Anatomía Patológica, Clínica Santa María. Santiago de Chile.

$\mathrm{K}$ ikuchi y Fujimoto y cols fueron los primeros en describir la enfermedad de Kikuchi-Fujimoto en Japón en 1972, en forma independiente ${ }^{1,2}$. El nombre anatomopatológico de esta entidad es linfadenitis histiocítica necrotizante. El

Correspondencia a: Dr. Patricio Gac E. Santos Dumont 999. Teléfonos: 9788000-2014607-94398414.

E mail: patriciogac@yahoo.com.ar primer caso afectando cabeza y cuello fue descrito por Gleeson en 1985. Esta entidad predomina en Asia, pero rápidamente se ha comunicado en otras áreas del mundo. Afecta usualmente a mujeres bajo los 30 años, pero puede presentarse también en varones. Su etiología es incierta. Se ha sugerido un proceso apoptótico mediado por linfocitos $\mathrm{T}$ CD8-positivos. Su factor causal también es desconocido, pero se cree que puede estar involucrada una etiología viral o autoinmune ${ }^{3,4}$. Se manifiesta 
generalmente como adenomegalias en la región cervical posterior, pero puede manifestarse como linfoadenopatía generalizada. A veces se acompaña de fiebre y neutropenia. Los hallazgos de laboratorio son escasos, pudiéndose encontrar cambios inespecíficos en el hemograma, como neutropenia ligera, lo que la hace fácilmente confundible con linfoma o lupus eritematoso sistémico, tanto clínica como histológicamente ${ }^{5}$. Puede simular también linfadenitis tuberculosa. El diagnóstico se logra mediante el análisis anatomopatológico. El curso clínico y el pronóstico son buenos, tratándose de una enfermedad autolimitada, con recuperación espontánea dentro de semanas a meses, sin secuelas. Si bien la enfermedad es bastante conocida dentro del entorno histopatológico, existen pocos reportes a nivel mundial, por lo que es una entidad bastante desconocida para el clínico en general. Es importante para el médico conocer esta entidad, pues habitualmente se presentará como un paciente con adenopatía única o múltiple, pensándose en patologías más comunes como los linfomas, sobre todo cuando el cuadro se ha acompañado de fiebre.

Caso clínico 1. Se presenta una mujer de 37 años que consultó por una masa submaxilar, dura, adherida, no dolorosa, de 4 meses de evolución. No existían antecedentes de fiebre o baja de peso.
Al examen físico destacaba una masa redondeada, dura, de bordes definidos de 2,5 cm de diámetro, adherido a planos profundos, no dolorosa, en la región submaxilar izquierda. Además se palpaba una adenopatía cervical posterior alta izquierda de 1,5 cm de diámetro. La ecografía cervical (Figura 1) describió por delante de la glándula submaxilar izquierda, una imagen hipoecogénica, ovalada, sólida de 16 × 9 x $14 \mathrm{~mm}$, planteándose la posibilidad de una adenopatía como primera posibilidad. El estudio de laboratorio, que incluyó hemograma con VHS, perfil bioquímico y pruebas de coagulación, fueron normales, encontrándose solamente leve anisocitosis y neutrófilos vacuolados poco frecuentes. El recuento absoluto de neutrófilos fue de $2,97 \times 10^{3} \mathrm{~mm}^{3}$, con $54 \%$ en la fórmula leucocitaria (recuento de leucocitos de $5,5 \times 10^{3} \mathrm{x}$ $\mathrm{mm}^{3}$ ). La LDH plasmática fue $217 \mathrm{U} / \mathrm{lt}$ (normal de 135-214). El planteamiento diagnóstico clínico inicial fue de una adenopatía cervical persistente, planteándose la posibilidad de una adenopatía inespecífica, linfoma o tumor primario de la glándula submaxilar izquierda. Se llevó a cabo una biopsia excisional. La apreciación del cirujano fue de una adenopatía en contacto con la submaxilar, no formando parte de ella. La biopsia contemporánea reportó una linfoadenitis inespecífica. El estudio histológico definitivo (Figura 2) demostró "linfonodo con arquitectura parcialmen-

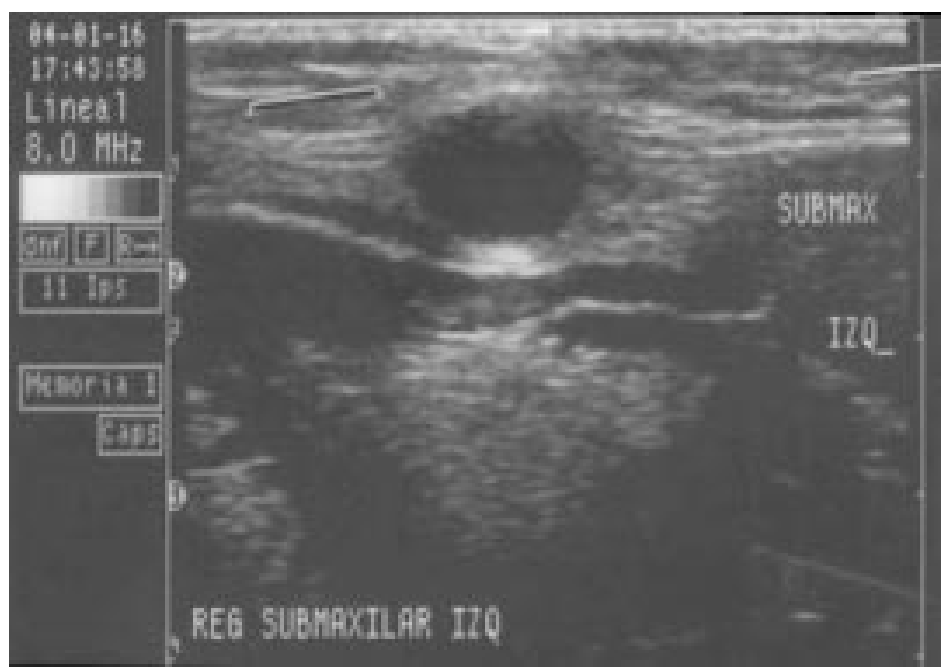

Figura 1. Ecografía cervical. Se observa una imagen hipoecogénica, ovalada, sólida de $16 \times 9 \times 14 \mathrm{~mm}$, planteándose la posibilidad de una adenopatía como primera posibilidad. 


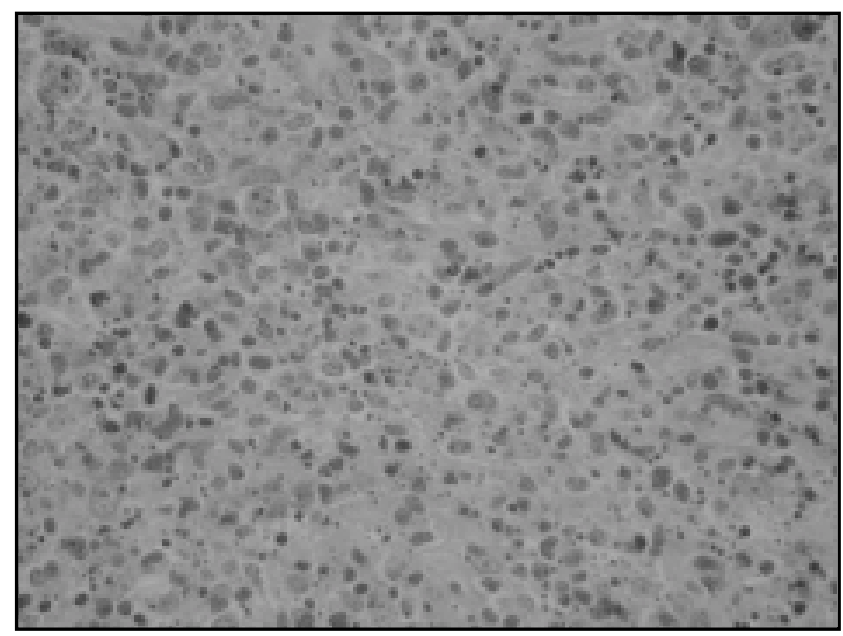

Figura 2. Tinción hematoxilina eosina 100X. Abundantes inmunoblastos, histiocitos y linfocitos pequeños.

te distorsionada debido a presencia de áreas claras y oscuras con algunos folículos linfoides periféricos de diferentes tamaños, con centros germinales normotípicos y efecto claro oscuro conservado. En las áreas claras interfoliculares se observaban zonas de necrosis constituidas por material eosinófilo, múltiples detritus celulares, con presencia de abundantes inmunoblastos, histiocitos y algunos linfocitos pequeños". Se realizaron las siguientes tinciones inmunohistoquímicas: CD 20 $(+)$ en linfocitos de los centros germinales y en escasos linfocitos pequeños de zonas interfoliculares, CD $45 \mathrm{RO}(+)$ en linfocitos pequeños interfoliculares, KAPPA y LAMBDA marcación policlonal, BCL 2 no satisfactoria, CD $15(-)$, CD 30 con tinción muy focal en algunos inmunoblastos, CD 10 no satisfactoria. El estudio informó finalmente linfoadenitis necrotizante histiocítica (enfermedad de Kikuchi). Actualmente con seguimiento de 26 meses, sin evidencia de recurrencia o secuelas.

Caso clínico 2. Mujer de 30 años, con cuadro de 2 meses de evolución de aumento de volumen cervical lateral izquierdo alto, indoloro, adherido, de aproximadamente $2,5 \mathrm{~cm}$ de diámetro. Resto del examen físico era normal. Sin fiebre o baja de peso. Hemograma y radiografía de tórax fueron normales. Fue manejada con antiinflamatorios no esteroidales (AINE), sin respuesta. En evaluación hematológica, se solicitó estudio con punción bajo ecografía. La citología era sospechosa de linfoma. Se decidió biopsia de la lesión. Se resecaron dos linfonodos de 1,0 y $1,6 \mathrm{~cm}$ de la región yúgulodigástrica izquierda, de aspecto inespecífico. El informe definitivo concluyó linfonodos de arquitectura parcialmente distorsionada, con expansión de áreas interfoliculares, en las que se observaba una población celular polimorfa que incluía células plasmáticas, polinucleares y macrófagos. La corteza estaba conservada. Se observaron macrófagos y focos de necrosis en áreas interfoliculares. (Figuras 3 y 4). En la tinción inmunohistoquímica se observó distribución normal de linfocitos CD 20 y CD 3. Marcador CD 68 positivo en los macrófagos y células histiocitoides. Hallazgos fueron compatibles con enfermedad de Kikuchi. Seguimiento clínico de 6 meses, sin evidencias de recurrencias ni secuelas.

Caso clínico 3. Paciente sexo femenino de 23 años, previamente sana. Presentó aumento de volumen cervical lateral izquierdo compatible clínicamente con adenopatía, de 3 semanas de evolución, indoloro, fijo a planos profundos de 2 $\mathrm{cm}$ de diámetro sin otros síntomas asociados. Se manejó con AINE sin buena respuesta. Se decidió biopsia de la lesión. Se resecó linfonodo de 1,5 $\mathrm{cm}$ de diámetro que al corte muestra áreas de necrosis. Histológicamente, se observó necrosis, sin supuración y sin granulomas. No se solicitó 


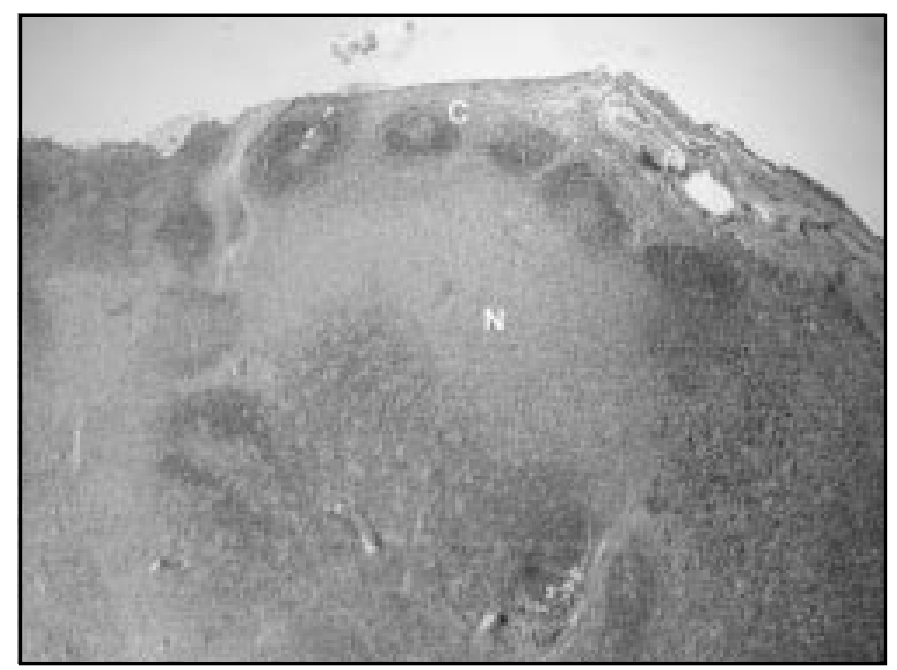

Figura 3. Tinción hematoxilina-eosina, aumento original de $40 \mathrm{x}$. $\mathrm{N}=$ necrosis y $\mathrm{C}$ =corteza linfonodo con arquitectura conservada y zona de necrosis subcortical.

Figura 4. Tinción hematoxilina-eosina, aumento original de 100x. $\mathrm{N}=$ necrosis. Se observa zona de necrosis en un folículo.

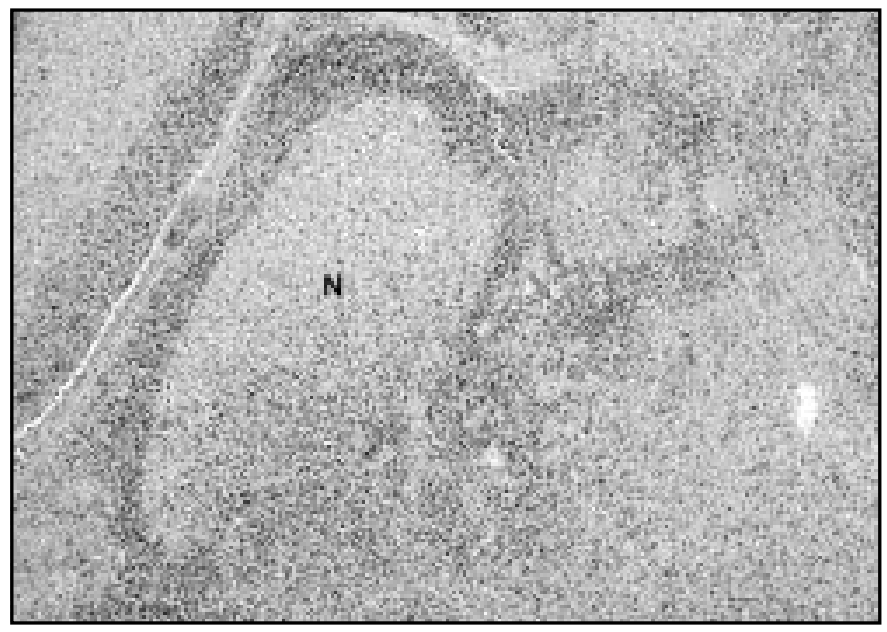

inmunohistoquímica por lo característico de la histología.

\section{Discusión}

La enfermedad de Kikuchi es una entidad clínica de muy baja frecuencia. En Chile sólo se han comunicado 3 casos, uno de ellos en una niña de 12 años tratada por 2 meses como amigdalitis, sin respuesta ${ }^{6-8}$.

La enfermedad afecta principalmente adultos menores de 40 años (19 meses a 75 años), con un promedio de edad de 21 años. Se ha reportado una relación mujer-hombre de 4:1 o 3:2, pero recientes trabajos de países orientales indican que podría haber sido sobrestimada y que la relación actual es cercana a 1:19.

La enfermedad tiene distribución mundial, con mayor prevalencia en la población asiática ${ }^{9}$.

Los pacientes con LHN se presentan habitualmente con linfoadenopatías, fiebre intermitente, malestar moderado, neutropenia leve. Raramente con manifestaciones cutáneas (máculas, pápulas 0 eritema urticarial o multiforme) y en $10 \%$ de los casos con hepato-esplenomegalia ${ }^{10-12}$. Menos fre- 
cuente es el compromiso neurológico, meningitis aséptica 0 ataxia cerebelosa ${ }^{13}$. A pesar que en $80 \%$ de las veces existen adenopatías generalizadas, la enfermedad es más llamativa por su localización cervical, siendo la ubicación posterior la predominante (65\% a $70 \%)$. La ubicación axilar es la segunda en frecuencia ${ }^{5}$. Los linfonodos comprometidos generalmente son indoloros.

La patogénesis no ha sido aún identificada. Se reporta que el interferón- $\gamma$, la interleukina- 6 o la muerte celular apoptótica podrían tener un rol en la patogénesis apoyando la hipótesis de una etiología viral o autoinmune ${ }^{3,4,9}$.

Algunos autores han indicado que existiría apoptosis celular, que estaría inducida por perforina o el ligando Fas, como el proceso principal en $\mathrm{LHN}^{14,15}$. Este hallazgo se vería en linfocitos y en histiocitos en los linfonodos afectados. Este proceso apoptótico sería responsable de la apariencia necrótica en los linfonodos. La perforina es una proteína citolítica específica para destruir células, es esencial en la inducción de la apoptosis y ha sido encontrada en abundancia en las células infiltrantes. En el estudio de Takakuwa y cols en 34 pacientes con LHN, se encontró que $82,4 \%$ de las células expresan perforina. Kato y cols encontraron que el ligando Fas estaba elevado y sugieren un posible rol en la patogénesis y en la presencia de síntomas; incluso podría actuar como un posible marcador de la enfermedad ${ }^{5,14,15}$. Algunos autores sugieren que la apoptosis celular Fas-inducida o perforina-inducida es comandada por linfocitos T CD8+ como el efector, y las células target e histiocitos jugarían un rol facilitador 3,16,17. Abe y cols demostraron la presencia de un receptor ligante de antígenos relacionados a cáncer expresado en células SISo (RCAS1) en macrófagos de linfonodos de pacientes con LHN. RCAS1 parece ser una proteína asociada a la apoptosis que induciría la apoptosis en células $\mathrm{T}$ activadas y en eritroblastos. El autor sugiere que la alta presencia de RCAS1 podría contribuir a la muerte de células T CD8+ dependiente de histio$\operatorname{citos}^{18}$.

Varios agentes infecciosos han sido postulados como su causa: Toxoplasma gondii, Yersinia enterocolítica, virus Epstein-Barr, herpes virus tipo 6 y 8, virus rubéola, paramyxovirus y virus de la parainfluenza, basados en resultados serológicos positivos $^{19}$. Sin embargo, los hallazgos histológi- cos de las linfadenitis asociadas a estos microorganismos claramente difieren de los encontrados en Kikuchi y varios estudios subsecuentes han fallado en demostrar esta hipótesis ${ }^{9}$.

Varios otros estudios han concluido que ni EBV ni herpesvirus 6,7 u 8 tendrían un rol en la patogénesis de Kikuchi, basado en el hecho de que la mayoría de los pacientes tienen serología negativa para estos agentes, y de ser positivos, el porcentaje de detección viral es menor que en los controles sanos ${ }^{9}$.

Un artículo tailandés sugiere asociación entre LHN y Mycobacterium szulgai basado en la coexistencia de hallazgos característicos en linfonodos y un cultivo positivo al Mycobacterium ${ }^{20}$. También se ha sugerido a Bartonella Henselae como posible agente etiológico en niños ${ }^{21}$.

Se han descrito traslocaciones cromosómicas, específicamente $\mathrm{t}(2: 16)$, asociadas a Kikuchi, anormalidades típicamente asociadas con condiciones linfoproliferativas malignas ${ }^{22}$.

Se cree que Kikuchi podría representar una respuesta inmune exagerada mediada por células $\mathrm{T}$ en personas genéticamente susceptibles en respuesta a una variedad de estímulos no específicos. Algunos genes HLA clase II son más frecuentes en pacientes con Kikuchi. En particular la incidencia de los alelos DPA1*01 y DPB1*0202 es significativamente mayor en pacientes con Kikuchi que en pacientes sanos. Estos genes son más frecuentes en personas asiáticas y esto podría explicar el patrón epidemiológico de la enfermedad $^{23}$.

El diagnóstico de la LHN se establece por biopsia excisional. El estudio citológico por aspiración con aguja fina puede establecerse sólo a veces, con una exactitud estimada de 56,3\%5,24. Habitualmente los métodos de imágenes como TC, RNM y ecografía, no ayudan en establecer el diagnóstico, aunque Miller y Pérez-Jaffe han descrito un patrón característico en CT y RNM consistente en pequeños linfonodos arracima$\operatorname{dos}^{25}$. Recientemente se ha descrito infiltración perinodal en $81,3 \%$ y aumento homogéneo del contraste nodal en $83,3 \%$ en un estudio que describe tomográficamente 1.196 linfonodos de 96 pacientes con LHN confirmada ${ }^{26}$.

Histológicamente la LHN se caracteriza por una colección de histiocitos y linfocitos rodeando áreas de necrosis. Los polimorfonucleares habi- 
tualmente están ausentes. Se han descrito los siguientes criterios: (1) áreas irregulares de necrosis eosinofílica en la corteza o paracorteza de los linfonodos, (2) fragmentos de material nuclear distribuidos de manera irregular en el área de necrosis, (3) ausencia de granulocitos y plasmocitos, (4) racimos de células T plasmocitoides y (5) numerosos inmunoblastos (linfocitos transformados, principalmente de fenotipo de células T) ${ }^{19}$.

Las células plasmocitoides predendríticas CD123 bright han sido descritas como un hallazgo histopatológico notable, pues podrían tener un rol en la patogénesis de LHN vía su migración desde la médula ósea a los linfonodos afectados, donde podrían producir grandes cantidades de interferón tipo I (habitualmente en respuesta a una infección viral), actuando como promotores de la respuesta de células $\mathrm{T}$ tipo $\mathrm{T}$-helper 1 con la consiguiente reacción inmune citotóxica ${ }^{9}$.

Kuo ha propuesto una clasificación de los hallazgos histológicos de la enfermedad de Kikuchi que la divide en 3 etapas: proliferativa, necrotizante y xantomatosa. La etapa proliferativa consiste en varios histiocitos, monocitos plasmocitoides y un número variable de células linfoides con fragmentos nucleares cariorrécticos y apoptosis eosinofílica. Si se agrega necrosis coagulativa se clasifica como necrotizante. Si existe predominio de histiocitos espumosos, el caso se clasifica como xantomatoso. Estas etapas podrían reflejar cambios histológicos progresivos en distintas fases de la enfermedad ${ }^{27}$.

El inmunofenotipo típico de LHN consiste en una predominancia de células $\mathrm{T}$ con predominancia de CD8+ sobre CD4+. La inmunohistoquímica compatible muestra CD68 y CD3 (+), CD20 (+/-) y CD30 (-). Los histiocitos expresan mieloperoxidasa y CD68.

El diagnóstico diferencial histopatológico debe establecerse con un gran número de enfermedades infecciosas, tuberculosis, sarcoidosis, procesos autoinmunes y enfermedades linfoproliferativas $^{10,28}$.

Debido a manifestaciones cutáneas observadas en LHN, como eritema facial y eritema multiforme, la relación de LHN y lupus eritematoso sistémico (LES) permanece en debate. Algunos autores sugieren que LHN es una manifestación temprana de $\mathrm{LES}^{29,30}$. Histopatológicamente LES muestra linfoadenopatías con hiperplasia folicular con granulocitos y células plasmáticas; hallazgos que no se encuentran en LHN. Además los resultados de estudios serológicos midiendo anticuerpos antinucleares, factor reumatoide y otros factores reumatológicos han resultado consistentemente negativos en estos pacientes, no apoyando la naturaleza autoinmune de la enfermedad ${ }^{31}$.

La asociación a LES ha sido descrita frecuentemente, sin embargo, no existen pruebas que confirmen esta relación ${ }^{9}$. También la ocurrencia simultánea de Kikuchi y linfadenitis en pacientes con fuga de silicona desde implantes mamarios ${ }^{32}$.

Dorfman y Berry han reportado LHN en pacientes VIH $(+)$, siendo importante en estos pacientes establecer el diagnóstico, tanto desde el punto de vista pronóstico, como para instaurar un determinado tratamiento ${ }^{33}$.

Es común la aproximación de LHN pensando en TBC linfonodal. Muchos de los casos descritos en la literatura están diagnosticados como TBC previo al estudio anatomopatológico, insistiéndose en su importancia al evitar instaurar tratamientos costosos y prolongados ${ }^{34}$. Otro diagnóstico diferencial importante es linfoma, existiendo casos descritos en la literatura de LHN tratados como linfoma ${ }^{10}$.

En el caso № 1, el enfrentamiento clínico inicial fue el de una adenopatía cervical persistente, planteándose la posibilidad de linfadenitis inespecífica, linfoma o tumor primario de la glándula submaxilar izquierda, por lo cual se planificó quirúrgicamente una biopsia linfonodal y la posibilidad de resección de submaxilar y posible vaciamiento de cuello.

LHN es una enfermedad autolimitada con un curso clínico benigno. La mayoría de los síntomas clínicos se resuelven de forma espontánea dentro de pocas semanas a 6 meses $^{5}$. Existen sin embargo reportes de recurrencia en la literatura, con un rango entre $3 \%$ y $4 \% 9,11,17$.

La experiencia del patólogo es fundamental para establecer el diagnóstico y considerar diagnósticos diferenciales, siendo de mucha utilidad las técnicas de inmunohistoquímica para descartar malignidad $^{6}$. Un estudio de 108 biopsias linfonodales mostró hasta 30\% de diagnóstico erróneo de linfoma ${ }^{33}$.

No existe terapia específica probada para la enfermedad y se recomienda la terapia sintomática. Se han utilizado antibióticos, AINE (siendo aspirina 
lo más utilizado), y corticoides (prednisona, prednisolona). Estos últimos han sido recomendados cuando no existe respuesta adecuada a AINE, resultando en una recuperación más temprana ${ }^{35}$.

Existe un caso de LHN asociado a artritis y sinovitis, sin respuesta a AINE (aspirina, indometacina), con respuesta favorable a prednisolona ${ }^{36}$.

Una publicación reciente describe tratamiento exitoso con cloroquina en un paciente con LHN mal diagnosticado como malaria. Un año después el paciente presentó recurrencia de la LHN y es tratado con hidroxicloroquina con una respuesta clínica a las $10 \mathrm{~h}$ luego de administrada la primera dosis $^{37}$.

Otras asociaciones descritas son LHN con tiroiditis de Hashimoto y carcinoma gástrico ${ }^{38,3}$.

Como complicación de la enfermedad se describen 4 casos de meningitis en la literatura, siendo la complicación más seria ${ }^{40}$.

\section{REFERENCIAS}

1. KIKUCHI M. Lymphadenitis showing focal reticulum cell hyperplasia with nuclear debris and phagocytosis. Nippon Ketsueki Gakkai Zasshi 1972; 35: 379-80.

2. Fujimoto Y, Kozima Y, Yamaguchi K. Cervical subacute necrotizing lymphadenitis. A new clinicopathological entity. Naika 1972; 376: 247-53.

3. Felgar RE, Furth EE, Wasik MA, Gluckman SJ, SALHANY KE. Histiocytic necrotizing lymphadenopathy (Kikuchi disease): In situ end-labeling, immunohistochemical, and serologic evidencesuppoting cytotoxic lymphocyte mediated apoptotic cell death. Mod Pathol 1997; 10: 231-41.

4. Kubota M, Tsukamoto R, Kurokawa K, Imai $T$, FuRusHo K. Elevated serum interferon gamma and interleukin-6 in patients with necrotizing lymphadenitis. Br J Hematol 1996; 95: 613-5.

5. Koybasi S, Saydam L, Gungen Y. Histiocytic Necrotizing Lymphadenitis of the Neck. Am J Otolaryngol 2003; 24: 344-7.

6. Tordecila J, Medina M, Ávila R. Enfermedad de Kikuchi-Fujimoto. Rev Chil Pediatr 2002; 73: 483-8.

7. Etcheverry R, Armas Cruz R, Martínez V. Linfoadenitis necrotizante subaguda (enfermedad de Kikuchi y Fujimoto). Rev Méd Chile 1990; 118: 431-6.
La mortalidad es rarísima, describiéndose sólo tres casos atribuidos a muerte por la enfermedad, una mujer de 38 años con falla cardíaca abrupta, la necropsia encontró múltiples focos de necrosis cardíaca; otro caso es un niño de 19 meses que fallece en el contexto de un síndrome febril, su necropsia muestra hallazgos histopatológicos típicos de LHN linfonodal y extranodal; por último se reportó un caso de LHN mortal en un paciente asiático trasplantado recientemente ${ }^{9,11}$.

En suma, LHN es una entidad clínica rara y desconocida para el clínico, de muy difícil diagnóstico. El médico debe conocer esta entidad y su asociación con otras patologías, asumiendo el cuadro como uno más de los diagnósticos posibles al enfrentarse a la biopsia de masas cervicales o axilares, teniendo en cuenta su curso autolimitado y excelente pronóstico, para no instaurar terapias prolongadas o costosas.

8. RoHMANN I, BENTJERODT R. Linfoadenitis necrotizante de Kikuchi: Histopatología en 2 casos. Rev Méd Chile 1988; 116: 929-33.

9. Bosch X, Guilabert A, Miquel R, Campo E. Enigmatic Kikuchi-Fujimoto disease: a comprehensive review. Am J Clin Pathol 2004; 122: 141-52.

10. Thongsuksai P, Kayasut K. Histiocytic necrotizing lymphadenitis. J Med Assoc Thai 1999; 82: 812-8.

11. Baumgartner BJ, Hewng ER Kikuchi disease: A case report and review of the literature. ENT J 2002; 81: 331-5.

12. Kosch M, Hausberg M, Barenbrock M, Rahn KH, KISTERS K. Histiocytic necrotizing lymphadenitis as a rare cause of cervical lymphadenopathy and fever. A case of biopsy proven recurrence over 19 years. Eur J Haematol 1999; 62: 282-3.

13. Debley JS, Rozansky DJ, Miler ML, Katz BZ, GREENE ME. Histiocytic necrotizing lymphadenitis with autoinmune phenomena and meningitis in a 14 year-old girl. Pediatrics 1996; 98: 130-3.

14. Krueger GR, Huetter ML, Rojo J, Romero M, CruzOrTiz H. Human herpes viruses HHV-4 (EBV) and HHV-6 in Hodkin's and Kikuchi's diseases and their relation to proliferation and apoptosis. Anticancer Res 2001; 21: 2155-61.

15. TaKakuwa T, OhNuma S, Koike J, Hoshikawa M, Korzum $\mathrm{H}$. Involvement of cell-mediated killing in 
apoptosis in histiocytic necrotizing lymphadenitis (Kikuchi-Fujimato Disease). Histopathology 1996; 28: 41-8.

16. Ohshima K, Shimazaki K, Kume T, Suzumiya J, Kanda M, KiкUCHi M. Perforin and Fas pathways of cytotoxic $\mathrm{T}$ cells in histiocytic necrotizing lymphadenitis. Histopathology 1998; 33: 471-8.

17. Ohshima K, Shimazaki K, Suzumiya J, Kanda M, Kumagawa M, Kikuchi M. Apoptosis of cytotoxic Tcells in histiocytic necrotizing lymphadenitis. Virchows Arch 1998; 433: 131-4.

18. Abe $Y$, Ohshima $K$, Nakashima M, Hara $K$, MatsushiMA T, CHOI I ET AL. Expression of apoptosisassociated protein RCAS1 in macrophages of histiocytic necrotizing lymphadenitis. Int J Hematol 2003; 77: 359-63.

19. Lin HC, Su CY, Huang CC, Hwang CF, Chien CY. Kikuchi's disease. A review and analysis of 61 cases. Otolaryngol Head Neck Surg 2003; 128: 650-3.

20. Maek-A-Nantawat W, Viriyavejakul P. Mycobacterium szulgai lymphadenitis mimicking Kikuchi's disease in Thailand. Southeast Asian J Trop Med Public Health 2001; 32: 537-40.

21. Chung JY, KIm SW, HaN TH, Lim SJ. Detection of the Bartonella henselae gene sequence in lymph nodes of children with Kikuchi's disease. Pediatrics 2005; 115: 1112.

22. Robertson K, Forsyth P, Batstone P, Levison D, GoodLAD J. Kikuchi's disease displaying a t(2:16) chromosomal translocation. J Clin Pathol 2007; 60: 433-5.

23. Tanaka T, Ohmori M, Yasunaga S, Ohshima $\mathrm{K}$, KIKUCHI M, SASAZUKI T. DNA typing of HLA class II genes (HLA-DR, -DQ and -DP) in Japanese patients with histiocytic necrotizing lymphadenitis (Kikuchi's disease). Tissue Antigens 1999; 54: 246-53.

24. Tong TR, Chan OW, Lee KC. Diagnosing Kikuchi disease on fine needle aspiration biopsy: a retrospective study of 44 cases diagnosed by cytology and 8 by histopathology. Acta Cytol 2001; 45: 953-7.

25. Miuer WT JR, Pérez-Jaffe LA. Cross-sectional imaging of Kikuchi disease. J Comput Assist Tomogr 1999; 23: 548-51.

26. Kwon SY, Kim TK, Kim YS, Lee KY, Lee NJ, Seol HY. CT Findings in Kikuchi Disease: Analysis of 96 Cases. AJNR Am J Neuroradiol 2004; 25: 1099-102.
27. Kuo T. Kikuchi's disease (histiocytic necrotizing lymphadenitis): a clinicopathologic study of 79 cases with an analysis of histologic subtypes, immunohistology, and DNAploidy. Am J Surg Pathol 1995; 19: 798-809.

28. Yoshino $T$, Mannami $T$, Ichimura $K$, Takenaka $K$, Nose S, Yamadori I et al. Two cases of histiocytic necrotizing lymphadenitis following diffuse large B-cell lymphoma. Hum Pathol 2000; 31: 1328-31.

29. Kaur S, Thami GP, Mohan H, Kanwar AJ. Kikuchi disease with facial rash and erythema multiforme. Pediatr Dermatol 2001; 18: 403-5.

30. Rakic L, Arrese JE, Thiry A, Pierard GE. Kikuchi Fujimoto lymphadenitis with cutaneous involvement. J Eur Acad Dermatol Venereol 1999; 13: 118-22.

31. Dorfman RF. Histiocytic necrotizing lymphadenitis of Kikuchi and Fujimoto [editorial]. Arch Pathol Lab Med 1987; 111: 1026-9.

32. Sever CE, Leith CP, Appenzehler J, Foucar K. Kikuchi's histiocytic necrotizing lymphadenitis associated with ruptured silicone breast implant. Arch Pathol Lab Med 1996; 120: 380-5.

33. Dorfman RF, BerRY GJ. Kikuchi's histiocytic necrotizing lymphadenitis: an analysis of 108 cases with emphasis on differencial diagnosis. Semin Diagn Pathol 1988; 5: 329-45.

34. Jayaraj SM, Lloyd J, Frosh AC, Patel KS. KikuchiFujimoto's syndrome masquerading as tuberculosis. J Laryngol Otol 1999; 113: 82-4.

35. Jang YJ, PARK KH, SeOK HJ. Management of Kikuch's disease using glucocorticoid. J Laryngol Otol 2000; 114: 709-11.

36. Graham LE. Kikuchi-Fujimoto disease and peripheral arthritis: A first! Ann Rheum Dis 2002; 61: 475.

37. Rezai K, Kuchipudi S, Chundi V, Ariga R, Loew J, Sha BE. Kikuchi-Fujimoto Disease: Hydroxychloroquine as a Treatment. Clin Infect Dis 2004; 39: 124-6.

38. Aqel N, Amr S, NajJAR M, Henry K. Kikuchi's lymphadenitis developing in a patient with mixed connective tissue disease and Hashimoto's Thyroiditis. Br J Rheumatol 1997; 36: 1236-8.

39. RadHi JM, SkinNideR L, McFadden A. Kikuchi's lymphadenitis and carcinoma of the stomach. J Clin Pathol 1997; 50: 530-1.

40. Sato Y, Kuno H, Oizum K. Histiocytic necrotizing lymphadenitis (Kikuchi's disease) with aseptic meningitis. J Neurol Sci 1999; 163: 187-91. 